\title{
Clinical characteristics and potential factors for recurrence of positive SARS-CoV-2 RNA in convalescent patients: a retrospective cohort study
}

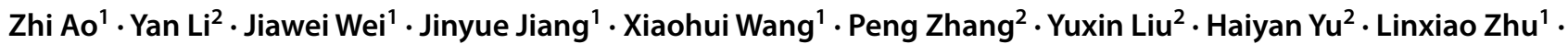

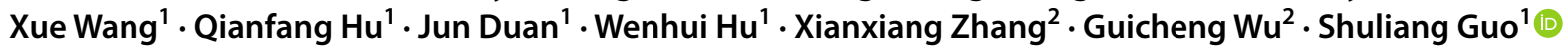

Received: 9 June 2020 / Accepted: 18 January 2021 / Published online: 4 February 2021

(c) The Author(s), under exclusive licence to Springer Nature Switzerland AG part of Springer Nature 2021

\begin{abstract}
Background The recurrence of positive SARS-CoV-2 RT-PCR is frequently found in discharged COVID-19 patients but its clinical significance remains unclear. The potential cause, clinical characteristics and infectiousness of the recurrent positive RT-PCR patients need to be answered.

Methods A single-centered, retrospective study of 51 discharged COVID-19 patients was carried out at a designated hospital for COVID-19. The demographic data, clinical records and laboratory findings of 25 patients with recurrent positive RT-PCR from hospitalization to follow-up were collected and compared to 26 patients with negative RT-PCR discharged regularly during the same period. Discharged patients' family members and close contacts were also interviewed by telephone to evaluate patients' potential infectiousness.

Results The titer of both IgG and IgM antibodies was significantly lower $(p=0.027, p=0.011)$ in patients with recurrent positive RT-PCR. Median duration of viral shedding significantly prolonged in patients with recurrent positive RT-PCR (36.0 days vs 9.0 days, $p=0.000$ ). There was no significant difference in demographic features, clinical features, lymphocyte subsets count and inflammatory cytokines levels between the two groups of patients. No fatal case was noted in two groups. As of the last day of follow-up, none of the discharged patients' family members or close contact developed any symptoms of COVID-19.

Conclusions Patients with low levels of IgG and IgM are more likely to have recurrent positive SARS-CoV-2 RT-PCR results and lead to a prolonged viral shedding. The recurrent positive of SARS-CoV-2 RT-PCR may not indicate the recurrence or aggravation of COVID-19. The detection of SARS-CoV-2 by RT-PCR in the patients recovered from COVID-19 is not necessarily correlated with the ability of transmission.
\end{abstract}

Keywords Coronavirus disease 2019 - RT-PCR · Viral shedding · Recurrent positive viral RNA

Zhi Ao, Yan Li and Jiawei Wei have contributed equally to the work.

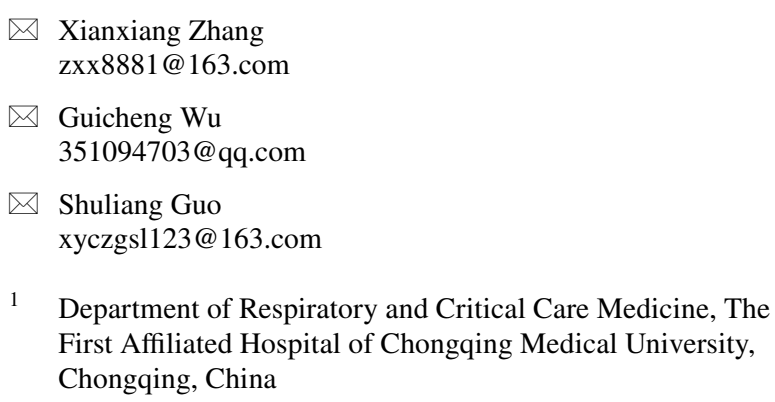

1 Department of Respiratory and Critical Care Medicine, The First Affiliated Hospital of Chongqing Medical University, Chongqing, China

2 Three Gorges Hospital of Chongqing University, Chongqing 404100, China

\section{Introduction}

An outbreak of coronavirus disease 2019 (COVID-19) caused by severe acute respiratory syndrome coronavirus 2 (SARS-CoV-2) was first reported in Wuhan, China in December 2019. Now it is effectively contained in China, but it has spread around the world and caused a global pandemic [1]. As a communicable disease caused by a novel virus, some clinical features and the course of COVID-19 are still not fully understood. At the very beginning of the outbreak, there were many reports on the diagnosis and treatment of COVID-19 patient, but the follow-up of the discharged patients has been ignored [2]. As the increase of discharged patients, some questions noticed in patients' 
recovery phase remain to be answered. Among them, the recurrence of positive SARS-CoV-2 RT-PCR received wide attention during the follow-up of the COVID-19 patients. Its clinical significance remains unclear [3]. Recent studies indicate that the presence of viral RNA in respiratory tract secretions may be related to the virus shedding and transmission [4-6], but there is still no consensus over the handling of the patients who have positive RT-PCR testing again after discharge. We conducted a single-centered, retrospective study of 51 discharged COVID-19 patients to determine the clinical significance, including the clinical outcomes and risk of transmission, of the recurrent positive RT-PCR and to further predict the potential factors for the recurrence of positive RT-PCR in the recovery phase.

\section{Methods}

\section{Study design and participants}

This retrospective study was carried out at Chongqing Three Gorges Hospital that is a designated hospital for the COVID19 patients. From Feb 23, 2020 to March 18, 2020, a total of 25 discharged COVID19 patients had recurrent positive RT-PCR during follow-up. Another 26 patients with consecutively negative results of RT-PCR discharged in the same period were randomly selected as controls. All the 51 patients enrolled in this study were confirmed COVID-19 cases according to the diagnostic criteria of the New Coronavirus Pneumonia Prevention and Control Program published by the National Health Commission of China [1]. All the patients were discharged between Feb 17, 2020 and Feb 24, 2020 after 12-19 days standard treatment according to the "Treatment Protocol for Novel Coronavirus InfectionInduced Pneumonia version 7 (trial)" and met the following criteria of hospital discharge in China: (1) normal body temperature for at least 3 consecutive days, (2) significant improvement with acute respiratory symptoms, (3) significant improvement compared with acute stage on chest CT images, (4) two consecutive negative results for SARSCoV-2 RNA from respiratory secretion swabs obtained at least $24 \mathrm{~h}$ apart. All the patients were required to be home quarantined for 14 days after discharge from hospital, and follow "The Guidelines for Home Quarantine" issued by WHO in 2020.

The follow-up for all these discharged patients started from Feb 23, 2020 to March 18, 2020, and all the patients were recalled from home at 3rd-9th day of home quarantine for the SARS-CoV-2 re-testing. Both cloacal and nasopharyngeal swab samples were collected every three days for RT-PCR detection of SARS-CoV-2 in the same way as they were in the hospital. The quarantine period for the patient who had a recurrent positive RT-PCR was prolonged until a negative RT-PCR result was obtained. This study was approved by the Ethical Committee of the First Affiliated Hospital of Chongqing Medical Hospital (approved number 20200601), and the requirement for informed consent was waived by the Ethics Commission for emergency infectious diseases.

\section{Data collection}

Patients' epidemiological data, demographic characteristics, clinical features, laboratory findings, chest CT from illness onset to follow-up were extracted from the electronic medical records of Chongqing Three Gorges Hospital and analyzed at the First Affiliated Hospital of Chongqing Medical Hospital. All information was obtained by using a standardized data collection form. To evaluate the potential infectiousness of the discharged patients, all the patients' family members and close contacts were interviewed by telephone at the last day of home quarantine. All the data were reviewed by two investigators independently to verify data accuracy.

\section{Laboratory procedures}

The laboratory diagnosis for all the patients was conducted in the Department of Medical laboratory of Chongqing University Three Gorges Hospital and the Wanzhou Center for Disease Control and Prevention by using the RT-PCR test kit (Coyotebio, Beijing, China) recommend by the Chinese Center for Disease Control and Prevention (CCDC). The test procedure was strictly in accordance with WHO guidelines for SARS-CoV-2 RT-PCR [7, 8]. Both cloacal and nasopharyngeal swab samples were collected for the RT-PCR test every three days during hospitalization and follow-up. The chest CT scan, the titer of anti-SARS-CoV-2 $\mathrm{IgG}$ and IgM by chemiluminescent immunoassay, and the count of lymphocyte subsets by flow cytometry was obtained from every patient. To characterize the correlation of the patients' immune status with the recurrence of positive RTPCR in the recovery phase, the patients' serum inflammatory cytokines (IL-2, IL-4, IL-5, IL-6, IL-1B, IL-8, IL-10, IL-12P70, IL-17, IFN- $\alpha$, IFN- $\gamma$, TNF- $\alpha$ ) level were measured and evaluated by using flow cytometry referring to the manufacturer's instructions.

\section{Statistical analysis}

Continuous variables were expressed as median (IQR) and compared with the Mann-Whitney U test. Categorical variables were expressed as number (\%) and compared by $\chi 2$ test or Fisher's exact test between positive and negative groups. A two-sided $\alpha$ of less than 0.05 was considered statistically 
significant. Statistical analyses were done using the IBM SPSS Statistics, version 23.0, unless otherwise indicated.

\section{Results}

\section{Demographic, clinical and laboratory data in hospitalization}

This study included 51 confirmed COVID-19 patients discharged from February17, 2020 to February 24, 2020, 25 , of whom 25 patients had recurrent positive RT-PCR after discharge. No significant difference in patients' age, gender, underlying diseases, known high-risk exposures or symptomes was observed between the two groups
(Table 1). Most patients were in the general status of illness severity $(40,78.4 \%)$ on admission, while $6(11.8 \%)$ patients were transferred to the ICU after admission because of respiratory failure (Table 1). The median duration of illness from onset (i.e., before admission) to discharge was 21.0 days (IQR 17.0-26.0) (Table 2). The median length of hospital stay was 14.0 days (IQR 12.0-18.0). The median duration of normal temperature and cough-free prior to discharge was 10.0 days (IQR 8.0-12.0) and 3.0 days (IQR 2.0-5.0), respectively. The median count of negative PCR results before discharge was twice (IQR 2-3; range, 2-8). No significant difference in clinical symptoms, severity of illness, duration of illness or length of hospital stay was observed between the two groups.

Table 1 Demographic, clinical features, laboratory data on admission

\begin{tabular}{|c|c|c|c|c|}
\hline & Total $(n=51)$ & Positive cases $(n=25)$ & Negative cases $(n=26)$ & $p$ value \\
\hline Age, years & $47.0(40.5-55.0)$ & $47.0(43.0-55.0)$ & $46.5(35.0-53.0)$ & 0.491 \\
\hline Sex & & & & 0.645 \\
\hline Female & $20(39.2 \%)$ & $9(36.0 \%)$ & $11(42.3 \%)$ & \\
\hline Male & $31(60.8 \%)$ & $16(64.0 \%)$ & $15(57.7 \%)$ & \\
\hline Underlying diseases & $10(19.6 \%)$ & $7(28.0 \%)$ & $3(11.5 \%)$ & 0.260 \\
\hline Exposure history & $43(84.3 \%)$ & $22(88.0 \%)$ & $21(80.8 \%)$ & 0.745 \\
\hline Fever (temperature $\geq 37.3^{\circ} \mathrm{C}$ ) & $37(72.5 \%)$ & $19(76.0 \%)$ & $18(69.2 \%)$ & 0.588 \\
\hline Cough & $29(56.9 \%)$ & $11(44.0 \%)$ & $18(69.2 \%)$ & 0.069 \\
\hline Fatigue & $7(13.7 \%)$ & $2(8.0 \%)$ & $5(19.2 \%)$ & 0.575 \\
\hline Disease severity status & & & & 0.280 \\
\hline Asymptomatic & $5(9.8 \%)$ & $4(16.0 \%)$ & $1(3.8 \%)$ & \\
\hline General & $40(78.4 \%)$ & $19(76.0 \%)$ & $21(80.8 \%)$ & \\
\hline Severe & $6(11.8 \%)$ & $2(8.0 \%)$ & $4(15.4 \%)$ & \\
\hline Lymphocyte count & $1038.88 \pm 479.18$ & $1017.35 \pm 376.01$ & $1027.90 \pm 425.47$ & 0.859 \\
\hline
\end{tabular}

Data are given as $n(\%)$ or median (IQR) or $\bar{x} \pm s$

Table 2 Clinical characteristics and laboratory data prior to discharge

\begin{tabular}{|c|c|c|c|c|}
\hline & Total $(n=51)$ & Positive cases $(n=25)$ & Negative cases $(n=26)$ & $p$ value \\
\hline Duration of normal temperature, days & $10.0(8.0-12.0)$ & $10.0(8.0-11.5)$ & $10.0(8.3-12.8)$ & 0.691 \\
\hline Duration of recovery from cough, days & $3.0(2.0-5.0)$ & $3.0(2.0-4.5)$ & $3.0(2.0-5.5)$ & 0.81 \\
\hline Time from illness onset to hospital discharge, days & $21.0(17.0-26.0)$ & $21.0(17.0-26.0)$ & $22.0(18.0-26.0)$ & 0.816 \\
\hline Length of hospital stay, days & $14.0(12.0-18.0)$ & $14.0(12.0-19.0)$ & $14.0(11.0-17.0)$ & 0.188 \\
\hline Negative PCR results count & $2(2-3)$ & $3(2-3)$ & $2(2-4)$ & 0.895 \\
\hline Lymphocyte count (normal range $1530-3700 / \mu \mathrm{L}$ ) & $1214.39 \pm 402.5$ & $1146.72 \pm 338.4$ & $1289.46 \pm 452.9$ & 0.243 \\
\hline T lymphocyte count (normal range $699-2540 / \mu \mathrm{L}$ ) & $873.55 \pm 290.31$ & $834.68 \pm 293.56$ & $910.92 \pm 287.86$ & 0.354 \\
\hline B lymphocyte count (normal range $90-660 / \mu \mathrm{L}$ ) & $126.00(92.50-169.50)$ & $126.00(92.00-156.00)$ & $131.00(93.25-176.75)$ & 0.553 \\
\hline $\mathrm{CD}^{+}$lymphocyte (normal range $410-1590 / \mu \mathrm{L}$ ) & $485.53 \pm 188.02$ & $454.60 \pm 179.34$ & $515.27 \pm 194.80$ & 0.253 \\
\hline $\mathrm{CD}^{+}$lymphocyte (normal range $190-1140 / \mu \mathrm{L}$ ) & $360.02 \pm 141.33$ & $349.80 \pm 151.87$ & $370.24 \pm 132.28$ & 0.614 \\
\hline $\mathrm{CD}^{+} / \mathrm{CD}^{+}$ & $1.34(1.03-1.76)$ & $1.33(1.00-1.77)$ & $1.35(1.14-1.67)$ & 0.720 \\
\hline
\end{tabular}

Data are given as $n(\%)$ or median (IQR) or $\bar{x} \pm s$ 


\section{Comparison between patients with re-detectable and undetectable viral RNA}

During follow-up, 27 (52.9\%) patients still presented mild cough while fever and fatigue completely relieved. Merely 2 patients showed lymphopenia with the lymphocyte count $0.86 \times 10^{9} / \mathrm{L}$ and $0.94 \times 10^{9} / \mathrm{L}$, respectively (normal range $1.1-3.2 \times 10^{9} / \mathrm{L}$ ). Ground-glass opacity on CT scan still remained in more than half of patients, mainly distributed in bilateral lobes (Table 3). There was no significant difference in imaging findings, lymphocyte count and levels of cytokines between the two groups (Tables 1, 2,3). For these patients who had recurrent positive RT-PCR, the median duration of viral shedding was 36.0 days (IQR 30.0-43.0) from diagnosis date, significantly longer compared to 9.0 days (IQR 7.0-11.0) for patients with negative viral RNA ( $p=0.000$, Fig. 1). Prolonged duration of quarantine after hospital discharge was consequently caused, showing 18.0 days (IQR 17.0-21.0) in positive patients and 10.0 days (IQR 6.0-12.0) in negative patients ( $p=0.000$, Fig. 1). There was no significant difference in patients' serum inflammatory cytokine levels between the two groups. The titer of

Table 3 Clinical features, laboratory data and chest CT scan in the follow-up

\begin{tabular}{|c|c|c|c|c|}
\hline & Total $(n=51)$ & Positive cases $(n=25)$ & Negative cases $(n=26)$ & $p$ value \\
\hline Fever (temperature $\geq 37.3^{\circ} \mathrm{C}$ ) & $0(0 \%)$ & $0(0 \%)$ & $0(0 \%)$ & \\
\hline Cough & $27(52.9 \%)$ & $11(44.0 \%)$ & $6(23.1 \%)$ & 0.113 \\
\hline Fatigue & $0(0 \%)$ & $0(0 \%)$ & $0(0 \%)$ & \\
\hline Lymphocyte count below the normal range & $2(3.9 \%)$ & $2(8 \%)$ & $0(0 \%)$ & 0.912 \\
\hline $\mathrm{IgG}, \mathrm{S} / \mathrm{CO}$ & $15.62(3.81-33.45)$ & $9.55(2.24-25.65)$ & $25.09(5.92-84.50)$ & 0.027 \\
\hline$\leq 10$ & $20(39.2 \%)$ & $13(52.0 \%)$ & $7(26.9 \%)$ & 0.110 \\
\hline$>10$ to $\leq 30$ & $17(33.3 \%)$ & $8(32.0 \%)$ & $9(34.6 \%)$ & \\
\hline$>30$ & $14(27.5 \%)$ & $4(16.0 \%)$ & $10(38.5 \%)$ & \\
\hline $\mathrm{IgM}, \mathrm{S} / \mathrm{CO}$ & $1.70(0.52-4.91)$ & $0.63(0.39-3.82)$ & $2.46(0.93-5.33)$ & 0.011 \\
\hline$\leq 1$ & $23(45.1 \%)$ & $16(64.0 \%)$ & $7(26.9 \%)$ & 0.027 \\
\hline$>1$ to $\leq 10$ & $20(39.2 \%)$ & $6(24.0 \%)$ & $14(53.8 \%)$ & \\
\hline$>10$ & $8(15.7 \%)$ & $3(18.0 \%)$ & $5(19.2 \%)$ & \\
\hline IL-2 (normal range $\leq 7.5 \mathrm{pg} / \mathrm{mL}$ ) & $0.00(0.00-0.02)$ & $0.00(0.00-0.06)$ & $0.00(0.00-0.01)$ & 0.206 \\
\hline IL-6 (normal range $\leq 5.4 \mathrm{pg} / \mathrm{mL}$ ) & $1.61(1.08-2.27)$ & $1.61(0.60-2.45)$ & $1.57(1.30-2.08)$ & 0.832 \\
\hline IL-8 (normal range $\leq 20.6 \mathrm{pg} / \mathrm{L}$ ) & $4.17(1.80-7.39)$ & $4.17(1.45-8.16)$ & $3.83(1.89-6.38)$ & 0.903 \\
\hline IL-1B(normal range $\leq 12.4 \mathrm{pg} / \mathrm{mL}$ ) & $0.16(0.00-0.94)$ & $0.00(0.00-0.84)$ & $0.49(0.00-0.96)$ & 0.456 \\
\hline IFN- $\gamma($ normal range $\leq 23.1 \mathrm{pg} / \mathrm{mL})$ & $1.51(0.68-2.92)$ & $1.12(0.54-2.97)$ & $1.80(1.12-2.84)$ & 0.550 \\
\hline During of viral shedding, days & $18.0(9.0-35.5)$ & $36.0(30.0-43.0)$ & $9.0(7.0-11.0)$ & 0.000 \\
\hline \multicolumn{5}{|l|}{ Imaging features } \\
\hline Consolidation & $3(5.9 \%)$ & $2(8.0 \%)$ & $1(3.8 \%)$ & 0.972 \\
\hline Ground-glass opacity & $33(64.7 \%)$ & $15(60.0 \%)$ & $18(69.2 \%)$ & 0.490 \\
\hline Bilateral infiltration & $32(62.7 \%)$ & $14(56.0 \%)$ & $18(59.2 \%)$ & 0.329 \\
\hline
\end{tabular}

Data are given as $n(\%)$ or median (IQR)

$S / C O$ signal/cut-off, $\downarrow$ below normal range, Ig immunoglobulin, $C T$ computed tomography, $I L$ interleukin, $I F N$ interferon

Fig. 1 Clinical conditions and duration of viral shedding in patients recovered from COVID-19 with different SARS-CoV-2 RNA testing results. Figure shows median duration of viral shedding and major symptoms and outcomes. COVID-19=coronavirus disease 2019. SARS-CoV-2=severe acute respiratory syndrome coronavirus 2

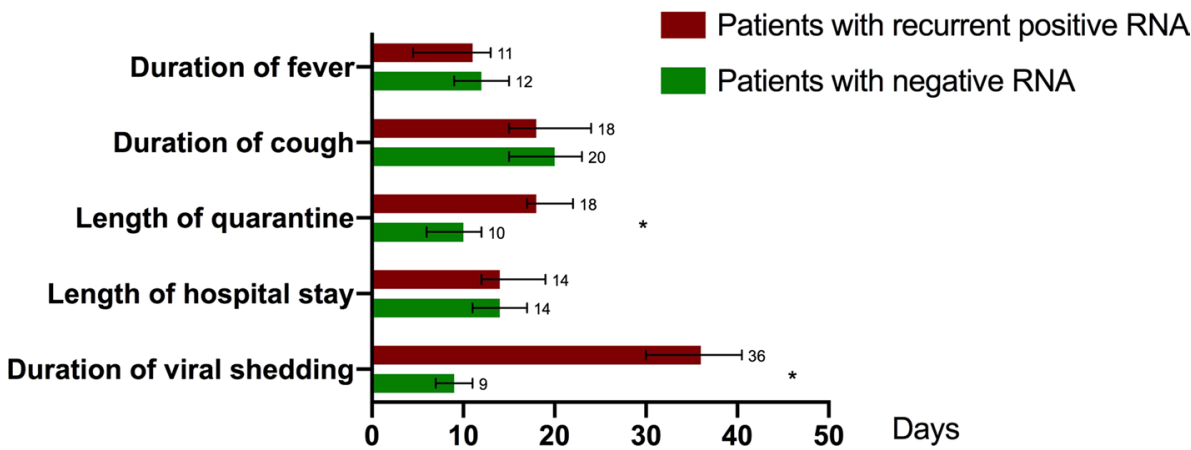


IgG and IgM antibody was the only one found significantly different among the immunological tests panel. The patients with recurrent positive RT-PCR had significantly lower IgG $(p=0.027)$ and $\operatorname{IgM}(p=0.011)$ antibody titer after discharge. No fatal case was noted in two groups during followup, and all the patients were released from quarantine.

\section{Infectiousness of discharged patients}

All discharged patients' family members and close contacts were interviewed by telephone. Although required, the instruction for home quarantine was not strictly followed by every discharged patient. An average of 3 family members had close contact with each discharged patient. 38 (74.5\%) of the patients were not allocated a separate room for home quarantine. 34 (66.7\%) of discharged patients wore a surgical mask in daily contact with their family members. Only 21 of discharged patients' utensils and clothes were disinfected routinely by using $75 \%$ ethanol $(19,37.3 \%)$ or household UV lamp $(2,4.3 \%)$. As of the last day of the home quarantine, none of the discharged patients' family members or close contacts developed any symptoms of COVID-19. Only 5 contacts reported insomnia, constipation and dizzy that are not typical symptoms of COVID-19 (Table 4).

\section{Discussion}

In this retrospective cohort study, a total of 51 patients including 25 cases with recurrent positive RT-PCR recovered from COVID-19 were enrolled. Patients with recurrent positive RT-PCR had lower antibody titers of IgG and IgM, longer viral shedding than patients with negative RT-PCR. Demographic, clinical characteristics, outcomes, lymphocyte subsets count and inflammatory cytokines levels were comparable between the two groups of patients. According to the Report of the WHO-China Joint Mission on Coronavirus Disease 2019, SARS-CoV-2 virus can initially be detected 1-2 days prior to symptom onset in upper respiratory tract samples and persist for 7-12 days in moderate cases and up to 2 weeks in severe cases [9]. But in our clinical practice the viral RNA can last up to 34 days by using RT-PCR method. Although the viral clearance is closely related to the host immune status and may vary from person to person [10], the significance of the viral shedding for transmission still has to be clarified. Fever, cough and fatigue are the most common symptoms in both groups as reported previously [11]. But none of these recurrent positive cases showed increased symptoms or deterioration in clinical conditions during follow-up after discharge, suggesting that the recurrent RT-PCR positive of SARS-CoV-2 may not indicate the recurrence or aggravation of COVID-19.

Nucleic acid-based methods including gene sequencing and RT-PCR are widely used for SARS-CoV-2 detection, however, a positive RT-PCR result can be also produced by non-infectious viruses present in the sample [12]. For some viruses like ZIKV, the viral RNA can persist in body fluid for a long time even after the infectious virus is cleared [13]. The instance of human ZIKV infection demonstrates that viral nucleic acid shedding in semen can be detectable in semen up to 181 days after symptom onset, but no infectious virus can be recovered from viral RNA-positive serum, urine, saliva or semen specimens [14]. The mechanisms of viral RNA persistence in the human host, the cellular reservoirs involved, as well as the mechanisms of viral clearance are still unknown, but at least limited evidence suggests that the presence of viral RNA is not exactly equivalent to the infectiousness. Therefore, the RT-PCR may not be the best option for the assessment of the infectiousness of the COVID-19 patient, even though the consecutive negative
Table 4 Details of home quarantine

\begin{tabular}{lllll}
\hline & Total $(n=51)$ & Positive cases $(n=25)$ & Negative cases $(n=26)$ & $p$ value \\
\hline Duration of home stay (day) & $5(3-9)$ & $4(2-9)$ & $6(3-8)$ & 0.482 \\
Family member contacts & $3(2-4)$ & $3(2-4)$ & $3(2-4)$ & 0.462 \\
Symptoms reported by contacts & $5(10.9 \%)$ & $2(9.0 \%)$ & $3(12.5 \%)$ & 0.918 \\
Types of home quarantine & & & & 0.687 \\
Separate room & $13(25.5 \%)$ & $7(28.0 \%)$ & $6(23.1 \%)$ & \\
Shared room & $38(74.5 \%)$ & $18(72.0 \%)$ & $20(76.9 \%)$ & 0.692 \\
Protective equipment & & & $18(69.2 \%)$ & 0.627 \\
$\quad$ Mask & $34(66.7 \%)$ & $16(64.0 \%)$ & $9(34.6 \%)$ & 0.509 \\
Medical alcohol & $19(37.3 \%)$ & $10(40.0 \%)$ & $2(8.3 \%)$ & - \\
Household UV lamp & $2(4.3 \%)$ & $0(0 \%)$ & - & \\
CT scans of contacts & - & - & - & \\
Viral RNA results contacts & - & - & & \\
\hline
\end{tabular}

Data are given as $n(\%)$ or median (IQR)

$U V$ ultraviolet, $C T$ computed tomography 
results are still required for discharge according to the latest treatment protocol of COVID-19. In China, the recurrent RTPCR positive patient is usually required to be quarantined until a negative RT-PCR result is obtained to prevent community transmission, but the viability of the SARS-CoV-2 detected by RT-PCR in these patients has not been proven by viral culture. The infectiousness of the recurrent RTPCR positive patient remains unclear. A previous study has reported that viable SARS-CoV could be isolated from stool and urine specimen more than 4 weeks after illness onset, which suggests potential infectious possibilities via excretions of convalescent individuals [15]. However, according to our findings, there is not enough evidence showing that the discharged patients with a recurrent positive RT-PCR could lead to the transmission of SARS-CoV-2 or adverse outcomes of their close contacts. In spite of this, since this study is limited to relatively small sample size and short duration of follow-up, cautions still need to be taken until more evidence is uncovered. It is also noteworthy that the false-negative of RT-PCR can frequently occur and lead to the miscarriage of patients' infectiousness [16], therefore the infectiousness of the COVID-19 patient cannot be ruled out by a negative RT-PCR result solely. Samples from multiple sites including throat, deep nasal cavity and cloaca should be tested repeatedly at different times to establish an authentic negative RT-PCR finding. Also, a negative RT-PCR result should be considered comprehensively in combine with additional antibody test, and radiographic findings to assure that infected patients have completely recovered and can be released from quarantine. Although the viral RNA detected by RT-PCR is not necessarily correlated with the ability of transmission, we still recommend that the recurrent positive RT-PCR patients should be closely followed up after discharge, and their infectiousness also needs to be assessed dynamically.

Previous studies have reported that the duration of SARSCoV-2 RNA persisted for 20 days in survivors and until death in non-survivors [17]. For Middle East respiratory syndrome coronavirus (MERS-CoV), ill patients shed the virus from respiratory tract secretions for much longer time than contacts [18]. Memish et al. attributed the difference to the severity of illness and comorbidities among ill cases [18]. Here, we found that the median duration of viral shedding was 18 days in all recovered patients. Among them, viral shedding duration was significantly longer in patients with recurred positive RNA than that in negative patients. In our study, positive cases continued to be quarantined until negative results were obtained. Thus, prolonged viral shedding contributed to longer duration of quarantine. Analysis of relationship between antibody response and viral load of SARS-CoV-2 was scarce. Victor et al. demonstrated that development of neutralizing antibody IgA could not effectively clear MERS-CoV from respiratory tract specimens
[19]. We speculated factors linked with prolonged viral shedding and found that it might be related to antibody response rather than cell-mediated immunity and inflammation response. The development of antibodies in serum might contribute to reduction of viral replication, leading to negative PCR testing results for SARS-CoV-2. Further research is needed to delineate the relationship between kinetics of viral loads and levels of antibodies.

Notably, this is the first study to demonstrate that patients with lower levels of IgG and IgM are more likely to have recurrent positive SARS-CoV-2 RNA testing results. Therefore, the results of the present study have contributed to the identification of the reason that patients who continue to improve in symptoms and chest CT scans have prolonged viral shedding. Considering the high detectable rate of positive viral RNA and potential infectiousness in convalescent patients, it is recommended that discharged patients be quarantined at home or in a designated site and underwent SARS-CoV-2 PCR testing. For individuals with recurrent positive RNA detection, they should be transferred to makeshift hospitals, nearby schools in suspension or hotels for at least 14 days of centralized isolation until viral RNA was undetectable. Given good prognosis of recovered patients, there is no need to prescribe additional antiviral medications unless clinical conditions progressed.

\section{Conclusions}

Patients with low levels of $\operatorname{IgG}$ and $\operatorname{IgM}$ are more likely to have recurrent positive SARS-CoV-2 RT-PCR results and lead to a prolonged viral shedding. The recurrent positive of SARS-CoV-2 RT-PCR may not indicate the recurrence or aggravation of COVID-19. The detection of SARS-CoV-2 by RT-PCR in the patients recovered from COVID-19 is not necessarily correlated with the ability of transmission, but we still recommend that the recurrent positive RT-PCR patients should be closely followed up after discharge, and their infectiousness also needs to be assessed dynamically.

Acknowledgements This research was funded by New coronavirus infection and prevention emergency scientific research project, Chongqing Education Board (KYYJ202006), Special Project for Emergency Research on 2019-nCoV Pneumonia, Chongqing Medical University (CQMU), and the fourth emergent science and technology special project on epidemic of novel coronavirus pneumonia, Chongqing Science and Technology Commission.

\section{Compliance with ethical standards}

Conflict of interest The authors declare no conflicts of interest.

Ethical approval This study was approved by the Ethical Committee of the First Affiliated Hospital of Chongqing Medical Hospital (approved 
number 20200601), and the requirement for informed consent was waived by the Ethics Commission for emergency infectious diseases.

\section{References}

1. China NHCotPsRo. Chinese management guideline for COVID19 (version 6.0). Feb 19, 2020. http://www.nhc.gov.cn/yzygj/ s7653p/202002/8334a8326dd94d329df351d7da8aefc2/files/b218c feb1bc54639af227f922bf6b817.pdf. Accessed 19 Feb 2020 (in Chinese).

2. Wang D, Hu B, Hu C, et al. Clinical characteristics of 138 hospitalized patients with 2019 novel coronavirus-infected pneumonia in Wuhan, China. JAMA. 2020;323:1061-9.

3. Lan L, Xu D, Ye G, et al. Positive RT-PCR test results in patients recovered from COVID-19. JAMA. 2020;323:1502-3.

4. Zou L, Ruan F, Huang M, et al. SARS-CoV-2 viral load in upper respiratory specimens of infected patients. N Engl J Med. 2020;382(12):1177-9.

5. Hoehl S, Rabenau H, Berger A, et al. Evidence of SARS-CoV-2 infection in returning travelers from Wuhan, China. N Engl J Med. 2020;382(13):1278-80.

6. Bai Y, Yao L, Wei T, et al. Presumed asymptomatic carrier transmission of COVID-19. JAMA. 2020;323:1406-7.

7. WHO. Laboratory testing for 2019 novel coronavirus (2019$\mathrm{nCoV}$ ) in suspected human cases. Interim guidance. 2020.

8. WHO. Clinical management of severe acute respiratory infection when novel coronavirus $(\mathrm{nCoV})$ infection is suspected. Interim guidance. 2020.

9. World Health Organization. Report of the WHO-China joint mission on coronavirus disease 2019 (COVID19). Geneva: WHO; 2020.

10. da Silveira MP, da Silva Fagundes KK, Bizuti MR, Starck E, Rossi $\mathrm{RC}$, de Resende ESDT. Physical exercise as a tool to help the immune system against COVID-19: an integrative review of the current literature. Clin Exp Med. 2020;29:1-14.
11. Gavriatopoulou M, Korompoki E, Fotiou D, et al. Organ-specific manifestations of COVID-19 infection. Clin Exp Med. 2020;20:493-506.

12. Cangelosi GA, Meschke JS. Dead or alive: molecular assessment of microbial viability. Appl Environ Microbiol. 2014;80(19):5884-91.

13. Duggal NK, Ritter JM, Pestorius SE, et al. Frequent Zika virus sexual transmission and prolonged viral RNA shedding in an immunodeficient mouse model. Cell Rep. 2017;18(7):1751-60.

14. Barzon L, Pacenti M, Franchin E, et al. Infection dynamics in a traveller with persistent. Shedding of Zika virus RNA in semen for six months after returning from Haiti to Italy, January 2016. Euro Surveill. 2016;21(32):30316.

15. Xu D, Zhang Z, Jin L, et al. Persistent shedding of viable SARS$\mathrm{CoV}$ in urine and stool of SARS patients during the convalescent phase. Eur J Clin Microbiol Infect Dis. 2005;24(3):165-71.

16. Xiao AT, Tong YX, Zhang S. False-negative of RT-PCR and prolonged nucleic acid conversion in COVID-19: rather than recurrence. J Med Virol. 2020;92:1755-6.

17. Zhou F, Yu T, Du R, et al. Clinical course and risk factors for mortality of adult inpatients with COVID-19 in Wuhan, China: a retrospective cohort study. Lancet. 2020;395(10229):1054-62.

18. Memish ZA, Assiri AM, Al-Tawfiq JA. Middle East respiratory syndrome coronavirus (MERS-CoV) viral shedding in the respiratory tract: an observational analysis with infection control implications. Int J Infect Dis. 2014;29:307-8.

19. Corman VM, Albarrak AM, Omrani AS, et al. Viral shedding and antibody response in 37 patients with middle east respiratory syndrome coronavirus infection. Clin Infect Dis. 2016;62(4):477-83.

Publisher's Note Springer Nature remains neutral with regard to jurisdictional claims in published maps and institutional affiliations. 